PROCEEDINGS OF THE

AMERICAN MATHEMATICAL SOCIETY

Volume 133, Number 3, Pages 699-709

S 0002-9939(04)07614-2

Article electronically published on September 29, 2004

\title{
QUANTUM BRUHAT GRAPH AND SCHUBERT POLYNOMIALS
}

\author{
ALEXANDER POSTNIKOV
}

(Communicated by John R. Stembridge)

\begin{abstract}
The quantum Bruhat graph, which is an extension of the graph formed by covering relations in the Bruhat order, is naturally related to the quantum cohomology ring of $G / B$. We enhance a result of Fulton and Woodward by showing that the minimal monomial in the quantum parameters that occurs in the quantum product of two Schubert classes has a simple interpretation in terms of directed paths in this graph.

We define path Schubert polynomials, which are quantum cohomology analogs of skew Schubert polynomials recently introduced by Lenart and Sottile. They are given by sums over paths in the quantum Bruhat graph of type $A$. The 3-point Gromov-Witten invariants for the flag manifold are expressed in terms of these polynomials. This construction gives a combinatorial description for the set of all monomials in the quantum parameters that occur in the quantum product of two Schubert classes.
\end{abstract}

\section{INTRODUCTION}

The famous Littlewood-Richardson coefficients are the structure constants of the cohomology ring of the Grassmannian in the basis of Schubert classes. It is an open question to give a combinatorial interpretation of the generalized LittlewoodRichardson coefficients for the flag manifold and, more generally, for the homogeneous space $G / P$. This article discusses the 3-point Gromov-Witten invariants, which are the structure constants of the small quantum cohomology ring. They extend the Littlewood-Richardson coefficients in an orthogonal "quantum" direction.

Fulton and Woodward [F-W] described the set of minimal monomials $q^{d}$ in the quantum parameters that occur in the quantum product $\sigma_{u} * \sigma_{v}$ of two Schubert classes in $\mathrm{QH}^{*}(G / P)$. We demonstrate that the problem of multiplying the Schubert classes in the quantum cohomology ring is naturally related to the study of paths in the quantum Bruhat graph from [BFP. This is the directed graph obtained by adding some "quantum" edges to the graph formed by covering relations in the Bruhat order. The quantum Bruhat graph encodes the terms that appear in the quantum Chevalley-Monk formula. We show, for $G / B$, that there is a unique minimal monomial $q^{d}$ that occurs in the quantum product $\sigma_{u} * \sigma_{v}$. This fact does not immediately follow from $[\mathrm{F}-\mathrm{W}]$. The minimal monomial $q^{d}$ is equal to the weight of any shortest directed path from $u$ to $w_{\mathrm{o}} v$ in the quantum Bruhat graph.

Received by the editors July 21, 2003 and, in revised form, November 20, 2003.

2000 Mathematics Subject Classification. Primary 05E05, 14N35, 14M15.

Key words and phrases. Quantum cohomology, flag manifold, Schubert polynomials.

The author was supported in part by NSF grant DMS-0201494.

(C)2004 American Mathematical Society Reverts to public domain 28 years from publication 
Lenart and Sottile [L-S] recently defined skew Schubert polynomials. The expansion coefficients of these polynomials in the basis of usual Schubert polynomials are equal to the generalized Littlewood-Richardson coefficients for the flag manifold. On the other hand, in [Po3, we introduced toric Schur polynomials, whose expansion coefficients in the basis of usual Schur polynomials are the Gromov-Witten invariants for the Grassmannian.

In this article, we put these two approaches together. We define the path Schubert polynomials, whose expansion coefficients in the basis of usual Schubert polynomials give the Gromov-Witten invariants for the flag manifold. The path Schubert polynomials can be defined as sums over directed paths in the quantum Bruhat graph on the symmetric group. The proof of the result on their Schubert expansion readily follows from a combination of the Cauchy identity, the elementary quantization rule from [FGP, and the quantum Pieri formula.

This construction implies a combinatorial description for the set of all monomials $q^{d}$ in the quantum parameters that occur, with nonzero coefficients, in the quantum product $\sigma_{u} * \sigma_{v}$ of two Schubert classes in the quantum cohomology of the flag manifold. A monomial $q^{d}$ occurs in $\sigma_{u} * \sigma_{v}$ if and only if there is a directed path of weight $q^{d}$ from $u$ to $w_{\mathrm{o}} v$ in the quantum Bruhat graph that satisfies a certain additional condition. This solves a problem posed by Fulton and Woodward [F-W] for the flag manifold.

The article consists of two parts: one related to the generalized flag manifold $G / B$, and the other concerned with the classical flag manifold $\mathrm{Fl}_{n}=\mathrm{SL}(n, \mathbb{C}) / B$. In Section 2, we discuss quantum cohomology of $G / B$ in a uniform Lie-theoretic setup. We enhance a result of Fulton and Woodward using the quantum Bruhat graph. In Section[3, we review some notions and results peculiar to the classical flag manifold. In Section 4, we use these combinatorial techniques to obtain stronger results for the quantum cohomology of $\mathrm{Fl}_{n}$.

\section{Quantum Cohomology of $G / B$}

In this section we discuss the quantum cohomology ring of the generalized flag manifold $G / B$. First, we briefly recall some general Lie-theoretic notation; see $[\mathrm{Hum}$ for more details. Then we recall a few facts related to Schubert classes and quantum cohomology, and formulate the quantum Chevalley-Monk formula; see [F-W]. This formula leads to the quantum Bruhat graph that was introduced in $[\mathrm{BFP}]$. We show that the minimal monomial $q^{d}$ that occurs in the quantum product of two Schubert classes has a simple interpretation in terms of directed paths in the quantum Bruhat graph.

Let $G$ be a simply connected complex semi-simple Lie group together with a Borel subgroup $B$. The homogeneous space $G / B$ is a compact complex manifold.

Let $\mathfrak{h}$ be the Cartan subalgebra in the Lie algebra $\mathfrak{g}$ of $G$, and let $\Phi \subset \mathfrak{h}^{*}$ be the root system associated with $G$. For a root $\alpha \in \Phi$, let $h_{\alpha} \in \mathfrak{h}$ denote the corresponding coroot in the dual root system $\Phi^{\vee}$. The Weyl group $W$ is generated by the reflections $s_{\alpha} \in \operatorname{End}\left(\mathfrak{h}^{*}\right), \alpha \in \Phi$, given by $s_{\alpha}: \lambda \mapsto \lambda-\lambda\left(h_{\alpha}\right) \alpha$. If $\langle\alpha \mid \beta\rangle$ is a $W$-invariant inner product on $\mathfrak{h}^{*}$, then $\mathfrak{h}$ can be identified with $\mathfrak{h}^{*}$ via this inner product and $h_{\alpha}=2 \alpha /\langle\alpha \mid \alpha\rangle$. The choice of Borel subgroup $B$ determines the set of positive roots $\Phi_{+} \subset \Phi$, the basis of simple roots $\alpha_{1}, \ldots, \alpha_{r}$ in the root system $\Phi$, and the basis of simple coroots $h_{\alpha_{1}}, \ldots, h_{\alpha_{r}}$ in the dual root system $\Phi^{\vee}$. The fundamental weights $\lambda_{1}, \ldots, \lambda_{r} \in \mathfrak{h}^{*}$ are defined as elements of the basis dual 
to $\left\{h_{\alpha_{1}}, \ldots, h_{\alpha_{r}}\right\}$, i.e., $\lambda_{i}\left(h_{\alpha_{j}}\right)=\delta_{i j}$. The Weyl group $W$ is generated by the set of Coxeter generators $s_{i}=s_{\alpha_{i}}$. The length $\ell(w)$ of an element $w \in W$ is the minimal number of generators $s_{i}$ in a decomposition for $w$.

The cohomology ring $\mathrm{H}^{*}(G / B)$ is a free $\mathbb{Z}$-module linearly generated by the Schubert classes $\sigma_{w}$ labeled by the elements $w \in W$ of the Weyl group. The Poincaré duality preserves the basis of Schubert classes:

$$
\int \sigma_{u} \cdot \sigma_{v}=\delta_{u, w_{\mathrm{o}} v} \quad \text { (Kronecker's delta). }
$$

Here and everywhere below $w_{\mathrm{o}}$ is the unique Weyl group element of maximal possible length, called the longest element.

The Schubert classes $\sigma_{s_{i}}$ generate the cohomology ring of $G / B$. According to Borel's theorem, the maps $\sigma_{s_{i}} \mapsto \lambda_{i}$ extend to the canonical isomorphism:

$$
\mathrm{H}^{*}(G / B, \mathbb{Q}) \simeq \operatorname{Sym}\left(\mathfrak{h}_{\mathbb{Q}}^{*}\right) / I_{W},
$$

where $\operatorname{Sym}\left(\mathfrak{h}_{\mathbb{Q}}^{*}\right)$ is the symmetric algebra of the $\mathbb{Q}$-span $\mathfrak{h}_{\mathbb{Q}}^{*} \subset \mathfrak{h}^{*}$ of the fundamental weights $\lambda_{i}$ and $I_{W}$ is the ideal in this algebra generated by $W$-invariant elements without constant term.

Let $\mathbb{Z}[q]=\mathbb{Z}\left[q_{1}, \ldots, q_{r}\right]$. The small quantum cohomology ring $\mathrm{QH}^{*}(G / B)$ of $G / B$ equals, as a $\mathbb{Z}[q]$-module, the tensor product $\mathrm{H}^{*}(G / B) \otimes_{\mathbb{Z}} \mathbb{Z}[q]$. Thus the Schubert classes $\sigma_{w}, w \in W$, form a $\mathbb{Z}[q]$-linear basis of $\mathrm{QH}^{*}(G / B)$. The multiplicative structure of the quantum cohomology is a deformation of the usual product in $\mathrm{H}^{*}(G / B)$. We will use the symbol "*" to denote the quantum product, i.e., the product in the quantum cohomology ring. The structure constants of the quantum cohomology are 3-point Gromov-Witten invariants:

$$
\sigma_{u} * \sigma_{v}=\sum_{w, d}\left\langle\sigma_{u}, \sigma_{v}, \sigma_{w_{\mathrm{o}} w}\right\rangle_{d} q^{d} \sigma_{w}
$$

where the sum is over $w \in W$ and $d=\left(d_{1}, \ldots, d_{r}\right) \in \mathbb{Z}_{>0}^{r}$, and $q^{d}=q_{1}^{d_{1}} \cdots q_{r}^{d_{r}}$. The Gromov-Witten invariants $\left\langle\sigma_{u}, \sigma_{v}, \sigma_{w_{\mathrm{o}} w}\right\rangle_{d}$ are nonnegative integers that count the numbers of certain rational curves in $G / B$. Their geometric definition implies that the quantum product is a commutative and associative operation.

For a root $\alpha \in \Phi_{+}$such that $h_{\alpha}=d_{1} h_{\alpha_{1}}+\cdots+d_{r} h_{\alpha_{r}}$, let $q^{h_{\alpha}}=q_{1}^{d_{1}} \cdots q_{r}^{d_{r}}$. Let us assume that the variables $q_{i}$ are of degree 2 . Thus $\operatorname{deg}\left(q^{h_{\alpha}}\right)=2\left|h_{\alpha}\right|$, where $\left|h_{\alpha}\right|=\left(\lambda_{1}+\cdots+\lambda_{r}\right)\left(h_{\alpha}\right)$ is the height of the coroot $h_{\alpha}$.

Let us define, for a positive root $\alpha \in \Phi_{+}$, the operator $\mathcal{T}_{\alpha}$ acting $\mathbb{Z}[q]$-linearly on the quantum cohomology ring $\mathrm{QH}^{*}(G / B)$ as

$$
\mathcal{T}_{\alpha}: \sigma_{w} \longmapsto\left\{\begin{array}{cl}
\sigma_{w s_{\alpha}} & \text { if } \ell\left(w s_{\alpha}\right)=\ell(w)+1, \\
q^{h_{\alpha}} \sigma_{w s_{\alpha}} & \text { if } \ell\left(w s_{\alpha}\right)=\ell(w)+1-2\left|h_{\alpha}\right|, \\
0 & \text { otherwise. }
\end{array}\right.
$$

The space $\mathrm{QH}^{*}(G / B)$ has the $\mathbb{Z}$-linear degree function $\operatorname{deg}\left(q^{d} \sigma_{w}\right)=2|d|+\ell(w)$. Then the $\mathcal{T}_{\alpha}$ are homogeneous operators on $\mathrm{QH}^{*}(G / B)$ of degree 1 .

The quantum cohomology $\mathrm{QH}^{*}(G / B)$ is generated, as an algebra over $\mathbb{Z}[q]$, by the Schubert classes $\sigma_{s_{i}}$. Thus the quantum product is uniquely determined by the following quantum Chevalley-Monk formula:

$$
\sigma_{s_{i}} * \sigma_{w}=\sum_{\alpha \in \Phi_{+}} \lambda_{i}\left(h_{\alpha}\right) \mathcal{T}_{\alpha}\left(\sigma_{w}\right)
$$


For root systems of type $A$, this formula was established in [FGP]. For the case of an arbitrary type it was found by Peterson [Pet] (unpublished). A more general version of the quantum Chevalley-Monk formula, for $\mathrm{QH}^{*}(G / P)$, was given and rigorously proved by Fulton and Woodward [F-W].

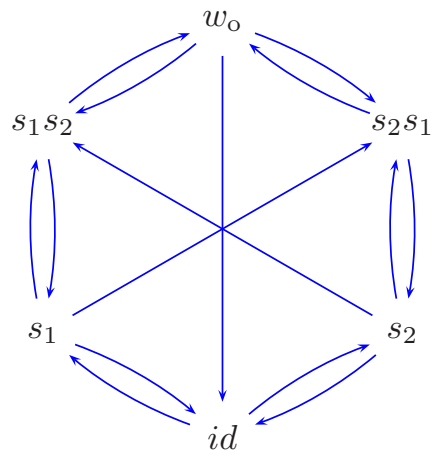

Figure 1. Quantum Bruhat graph $\Gamma_{\Phi}$ of type $A_{2}$

Motivated by the quantum Chevalley-Monk formula (3), let us define the quantum Bruhat graph $\Gamma_{\Phi}$ as the following directed graph on the Weyl group elements $w \in W$ with weighted edges. Two elements $u, v \in W$ are connected by a directed edge $e: u \rightarrow v$ if and only if $v=u s_{\alpha}$ and one of the following two conditions is satisfied:

$$
\ell(v)=\ell(u)+1 \quad \text { or } \quad \ell(v)=\ell(u)+1-2\left|h_{\alpha}\right| .
$$

If $\ell(v)=\ell(u)+1$, then the weight of edge $e$ equals 1 , and if $\ell(v)=\ell(u)+1-2\left|h_{\alpha}\right|$, then the weight of edge $e$ equals $q^{h_{\alpha}}$. The weight of a directed path in the graph $\Gamma_{\Phi}$ is the product of the weights of its edges.

The graph $\Gamma_{\Phi}$ was investigated in $[\mathrm{BFP}]$ and, for type $A$, in $[\mathrm{P} 02$. The upward edges in $\Gamma_{\Phi}$, i.e., the edges that increase the length by 1 , are exactly the covering relations in the Bruhat order on the Weyl group $W$. The downward edges correspond to additional "quantum" terms that appear in the quantum Chevalley-Monk formula (3). This is why we call $\Gamma_{\Phi}$ the quantum Bruhat graph.

Let us say that a directed path in $\Gamma_{\Phi}$ from $u$ to $v$ is shortest if it has the minimal possible length among all directed paths from $u$ to $v$.

Lemma 1. Let $u, v \in W$ be any two Weyl group elements. (1) There exists a directed path from $u$ to $v$ in the graph $\Gamma_{\Phi}$. (2) All shortest paths from u to $v$ have the same weight $q^{d_{\min }}$. (3) The weight of any path from $u$ to $v$ is divisible by $q^{d_{\min }}$.

This lemma says that, for any $u, v \in W$, there is a well-defined minimal degree $d_{\min }=d_{\min }(u, v) \in \mathbb{Z}_{\geq 0}^{r}$ such that $q^{d_{\min }}$ is the minimal possible weight of a directed path from $u$ to $v$. The lemma easily follows from results of [BFP].

Proof. Suppose that $a$ covers $b$ in the weak Bruhat order, i.e., $a, b \in W, a=b s_{i}$, and $\ell(a)=\ell(b)+1$. Then both directed edges $a \rightarrow b$ and $b \rightarrow a$ are present in the quantum Bruhat graph $\Gamma_{\Phi}$. Thus any two Weyl group elements $u$ and $v$ can be connected by a directed path in $\Gamma_{\Phi}$. 
According to [BFP, Lemma 6.7], for a path $a \rightarrow b \rightarrow c$ of length 2 in the graph $\Gamma_{\Phi}$ with $a \neq c$, there exists a unique path $a \rightarrow b^{\prime} \rightarrow c$ such that $b^{\prime} \neq b$. The proof of Theorem 6.4 in $\mathrm{BFP}$ implies that any two shortest paths from $u$ to $v$ can be obtained from each other by applying several switches of this type to pairs of consecutive edges in a path. Moreover, any path from $u$ to $v$ can be reduced to a shortest path by applying a sequence of such switches and cancellations of pairs of opposite edges $a \rightarrow b \rightarrow a$; see [BFP, Section 6].

These switches of pairs of consecutive edges in a path preserve its weight. Indeed, it is enough to verify the statement only for 3 types $A_{2}, B_{2}$, and $G_{2}$ of rank 2 root systems (cf. proof of BFP, Lemma 6.7]), which can be easily done by a direct observation. Thus all shortest paths from $u$ to $v$ have the same weight $q^{d_{\min }}$, and the weight of any path from $u$ to $v$ is divisible by $q^{d_{\min }}$.

Let $\ell(u, v)$ be the length of a shortest path from $u$ to $v$ in the graph $\Gamma_{\Phi}$.

Theorem 2. Fix two Weyl group elements $u, v \in W$. For any $w \in W$, the coefficient of $\sigma_{v}$ in $\sigma_{u} * \sigma_{w}$ is divisible by $q^{d_{\min }(u, v)}$. There exists $w \in W$ such that the coefficient of $\sigma_{v}$ in $\sigma_{u} * \sigma_{w}$ equals $q^{d_{\min }(u, v)}$ times a nonzero integer. Moreover, for any such $w$, we have $\ell(w)=\ell(u, v)$.

Proof. Let $S=\sigma_{s_{1}}+\ldots+\sigma_{s_{r}}$. The expression $S^{* l}=S * \cdots * S$ expands as a positive integer combination of the terms $q^{d} \sigma_{w}$. For any $w \in W$ of length $\ell(w)=l$, the expansion of $S^{* l}$ contains the class $\sigma_{w}$ with a strictly positive integer coefficient (without any quantum parameters $q_{i}$ ). According to the quantum Chevalley-Monk formula (3), the coefficient of $\sigma_{v}$ in $S^{* l} * \sigma_{u}$ is given by the sum over directed paths from $u$ to $v$, and each path comes with its weight times some positive integer. By Lemma 1, all these terms, including the contribution of $\sigma_{w} * \sigma_{u}$, should be divisible by $q^{d_{\min }(u, v)}$. This proves the first claim of the theorem.

On the other hand, suppose that $l=\ell(u, v)$. Then there exists at least one path from $u$ to $v$ of length $l$. By Lemma 1 and the quantum Chevalley-Monk formula (3), the coefficient of $\sigma_{v}$ in $S^{* l} * \sigma_{u}$ is equal to $q^{d_{\min }(u, v)}$ times a strictly positive integer. The expansion of $S^{* l}$ in the basis of Schubert classes may also contain terms $q^{d} \sigma_{w^{\prime}}$ with nonzero $d$. For all such terms, we have $\ell\left(w^{\prime}\right)=l^{\prime}<l$ because $l=\operatorname{deg}\left(S^{* l}\right)=\operatorname{deg}\left(q^{d} \sigma_{w^{\prime}}\right)=2|d|+l^{\prime}$. Thus $\sigma_{w^{\prime}}$ appears with a strictly positive coefficient in the expansion of $S^{* l^{\prime}}$. But, the coefficient of $\sigma_{v}$ in $S^{* l^{\prime}} * \sigma_{u}$ is 0 because there are no paths from $u$ to $v$ of length $l^{\prime}<l$. Thus the terms $q^{d} \sigma_{w^{\prime}}$ in $S^{* l}$ cannot make any contribution to the (nonzero) coefficient of $\sigma_{v}$ in $S^{* l} * \sigma_{u}$.

This means that there exists at least one term Const $\cdot \sigma_{w}$ in the expansion of $S^{* l}$ with $\ell(w)=\ell(u, v)$ that does make a contribution to the coefficient of $\sigma_{v}$ in $S^{* l} * \sigma_{u}$. In other words, the coefficient of $\sigma_{v}$ in $\sigma_{w} * \sigma_{u}$ equals $q^{d_{\min }(u, v)}$ times a nonzero integer, which proves the second claim of the theorem.

The next claim strengthens a result due to Fulton and Woodward [F-W] Theorem 9.1]. Let us say that a monomial $q^{d}$ occurs in the quantum product $\sigma_{u} * \sigma_{v}$ of two Schubert classes if there exists $w \in W$ such that the Gromov-Witten invariant $\left\langle\sigma_{u}, \sigma_{v}, \sigma_{w}\right\rangle_{d}$ is nonzero. Let us say that $q^{d}$ is a minimal monomial that occurs in $\sigma_{u} * \sigma_{v}$ if (1) $q^{d}$ occurs in $\sigma_{u} * \sigma_{v}$, and (2) any monomial strictly dividing $q^{d}$ does not occur in $\sigma_{u} * \sigma_{v}$. Theorem 2 can be equivalently reformulated, as follows.

Corollary 3. For any pair $u, v \in W$, the monomial $q^{d_{\min }\left(u, w_{\mathrm{o}} v\right)}$ is the unique minimal monomial that occurs in the quantum product $\sigma_{u} * \sigma_{v}$. 
Note that Fulton and Woodward [F-W] described the set of minimal monomials that occur in $\sigma_{u} * \sigma_{v}$, but their construction does not immediately imply that this set consists of a single element.

It would be interesting to describe all monomials $q^{d}$ that occur in a quantum product $\sigma_{u} * \sigma_{v}$. Theorem 2 suggests that such monomials $q^{d}$ should be weights of paths from $u$ to $w_{\mathrm{o}} v$ in the graph $\Gamma_{\Phi}$ that satisfy certain additional conditions. In the following sections we describe such a class of paths for type $A$ root systems.

\section{Flag MANifold and Schubert POlynomials}

There are some notions and results related to (quantum) cohomology, which are peculiar for type $A$. In this section we discuss this type $A$ theory, which includes the Cauchy identity for Schubert polynomials (see $[\mathrm{Mac}]$ ), the elementary quantization rule, and the quantum Pieri operators (see [FGP Po1] for more details).

For type $A_{n-1}$, the homogeneous space $G / B$ is the complex flag manifold $\mathrm{Fl}_{n}=$ $\mathrm{SL}(n, \mathbb{C}) / B$. The corresponding Weyl group is the symmetric group $W=S_{n}$ of order $n$ permutations. Thus the Schubert classes $\sigma_{w}$, which form a $\mathbb{Z}$-basis of the cohomology $\mathrm{H}^{*}\left(\mathrm{Fl}_{n}\right)$, are labeled by permutations $w \in S_{n}$.

In this case, Borel's theorem (1) implies that the cohomology ring is isomorphic to the following quotient of the polynomial ring:

$$
\mathrm{H}^{*}\left(\mathrm{Fl}_{n}\right) \simeq \mathbb{Z}\left[x_{1}, \ldots, x_{n}\right] /\left\langle e_{1}, \ldots, e_{n}\right\rangle,
$$

where $e_{i}=e_{i}\left(x_{1}, \ldots, x_{n}\right)$ are the elementary symmetric polynomials.

Lascoux and Schützenberger [LSc], using constructions of Bernstein-GelfandGelfand and Demazure, defined the Schubert polynomials $\mathfrak{S}_{w}(x)=\mathfrak{S}_{w}\left(x_{1}, \ldots, x_{n}\right)$ in the polynomial ring $\mathbb{Z}\left[x_{1}, \ldots, x_{n}\right]$, which are particularly nice polynomial representatives of the Schubert classes $\sigma_{w}$. These polynomials have nonnegative integer coefficients and are stable under the standard embedding $S_{n} \hookrightarrow S_{n+1}$. Another important property of the Schubert polynomials is the following Cauchy identity (see $\underline{\mathrm{Mac}})$ :

$$
\prod_{i+j \leq n}\left(x_{i}+y_{j}\right)=\sum_{w \in S_{n}} \mathfrak{S}_{w}\left(x_{1}, \ldots, x_{n}\right) \cdot \mathfrak{S}_{w w_{\mathrm{o}}}\left(y_{1}, \ldots, y_{n}\right) .
$$

For type $A_{n-1}$, the longest permutation in $S_{n}$ is given by $w_{\mathrm{o}}=n \ldots 21$.

The left-hand side of (5) is the double Schubert polynomial $\mathfrak{S}_{w_{\mathrm{o}}}(x,-y)$. It can be expanded in terms of elementary symmetric polynomials as

$$
\prod_{i+j \leq n}\left(x_{i}+y_{j}\right)=\prod_{k=1}^{n-1} \sum_{i=0}^{k} y_{n-k}^{k-i} e_{i}\left(x_{1}, \ldots, x_{k}\right) .
$$

As a linear space, the quantum cohomology ring $\mathrm{QH}^{*}\left(\mathrm{Fl}_{n}\right)$ of the flag manifold equals $\mathrm{H}^{*}\left(\mathrm{Fl}_{n}\right) \otimes \mathbb{Z}[q]$, where $\mathbb{Z}[q]=\mathbb{Z}\left[q_{1}, \ldots, q_{n-1}\right]$.

For type $A_{n-1}$, the definition (21) of the operators $\mathcal{T}_{\alpha}$ can be written as follows. For $1 \leq i<j \leq n$, let $\mathcal{T}_{i j}$ be the operator that acts $\mathbb{Z}[q]$-linearly on the quantum cohomology $\mathrm{QH}^{*}\left(\mathrm{Fl}_{n}\right)$ by the formula

$$
\mathcal{T}_{i j}: \sigma_{w} \longmapsto\left\{\begin{array}{cl}
\sigma_{w s_{i j}} & \text { if } \ell\left(w s_{i j}\right)=\ell(w)+1, \\
q_{i j} \sigma_{w s_{i j}} & \text { if } \ell\left(w s_{i j}\right)=\ell(w)+1-2(j-i), \\
0 & \text { otherwise, }
\end{array}\right.
$$


where $\ell(w)$ denote the length of the permutation $w, s_{i j} \in S_{n}$ is the transposition of $i$ and $j$, and $q_{i j}=q_{i} q_{i+1} \cdots q_{j-1}$. The Coxeter generators of $S_{n}$ are the adjacent transpositions $s_{k}=s_{k+1}, k=1, \ldots, n-1$. The quantum Chevalley-Monk formula (3) specializes to the following formula proved in [FGP]:

$$
\sigma_{s_{k}} * \sigma_{w}=\sum_{i \leq k<j} \mathcal{T}_{i j}\left(\sigma_{w}\right)
$$

for any $w \in S_{n}$ and $k=1, \ldots, n-1$.

Let us define an involution $\omega$ on the quantum cohomology ring $\mathrm{QH}^{*}\left(\mathrm{Fl}_{n}\right)$ by setting $\omega: f\left(q_{1}, \ldots, q_{n-1}\right) \mapsto f\left(q_{n-1}, \ldots, q_{1}\right)$ and

$$
\omega: q^{d} \sigma_{w} \mapsto \omega\left(q^{d}\right) \sigma_{w_{\mathrm{o}} w w_{\mathrm{o}}},
$$

and extending it by linearity. An easy observation shows that the quantum Chevalley-Monk formula (8) is invariant under $\omega$. Thus $\omega$ is an automorphism of $\mathrm{QH}^{*}\left(\mathrm{Fl}_{n}\right)$.

Let $e_{i}^{(k)}=\sigma_{(k-i+1, \ldots, k, k+1)}$ and $h_{i}^{(k)}=\sigma_{(k+i, \ldots, k+1, k)}$ be the Schubert classes represented in (44) by the elementary and complete homogeneous symmetric functions in the first $k$ variables: $e_{i}\left(x_{1}, \ldots, x_{k}\right)=\mathfrak{S}_{(k-i+1, \ldots, k+1)}$ and $h_{i}\left(x_{1}, \ldots, x_{k}\right)=$ $\mathfrak{S}_{(k+i, \ldots, k)}$. Here we use cycle notation for permutations: $(k-i+1, \ldots, k+1)=$ $s_{k-i+1} \cdots s_{k-1} s_{k}$ and $(k+i, \ldots, k)=s_{k+i-1} \cdots s_{k+1} s_{k}$. The involution $\omega$ on $\mathrm{H}^{*}\left(\mathrm{Fl}_{n}\right)$ switches these two families of Schubert classes $e_{i}^{(k)} \stackrel{\omega}{\longleftrightarrow} h_{i}^{(n-k)}$.

Theorem 1.1 from FGP is equivalent to saying that the quantum product of the Schubert classes $e_{i_{1}}^{(1)}, e_{i_{2}}^{(2)}, \ldots, e_{i_{n-1}}^{(n-1)}$ in the ring $\mathrm{QH}^{*}\left(\mathrm{Fl}_{n}\right)$ is exactly the same as the classical product of these Schubert classes in $\mathrm{H}^{*}\left(\mathrm{Fl}_{n}\right)$ :

$$
e_{i_{1}}^{(1)} * e_{i_{2}}^{(2)} * \cdots * e_{i_{n-1}}^{(n-1)}=e_{i_{1}}^{(1)} \cdot e_{i_{2}}^{(2)} \cdots \cdots e_{i_{n-1}}^{(n-1)} .
$$

We call this identity the elementary quantization rule. Applying the involution $\omega$ to both sides, we get a similar statement for the $h_{i}^{(k)}$ :

$$
h_{i_{1}}^{(1)} * h_{i_{2}}^{(2)} * \cdots * h_{i_{n-1}}^{(n-1)}=h_{i_{1}}^{(1)} \cdot h_{i_{2}}^{(2)} \cdots \cdots h_{i_{n-1}}^{(n-1)} .
$$

Let us define the quantum Pieri operators $\mathcal{E}_{i}^{(k)}$ and $\mathcal{H}_{i}^{(k)}$, acting on the quantum cohomology $\mathrm{QH}^{*}\left(\mathrm{Fl}_{n}\right)$, as

$$
\begin{aligned}
& \mathcal{E}_{i}^{(k)}=\sum \mathcal{T}_{a_{1} b_{1}} \cdots T_{a_{i} b_{i}}, \\
& \mathcal{H}_{i}^{(k)}=\sum \mathcal{T}_{c_{1} d_{1}} \cdots T_{c_{i} d_{i}},
\end{aligned}
$$

where the first sum is over $a_{1}, \ldots, a_{i}, b_{1}, \ldots, b_{i}$ such that

$$
a_{1}, \ldots, a_{i} \leq k<b_{1} \leq \cdots \leq b_{i} \text { and } a_{1}, \ldots, a_{i} \text { are distinct; }
$$

and the second sum is over $c_{1}, \ldots, c_{i}, d_{1}, \ldots, d_{i}$ such that

$$
c_{1} \leq \cdots \leq c_{i} \leq k<d_{1}, \ldots, d_{i} \text { and } d_{1}, \ldots, d_{i} \text { are distinct. }
$$

In [Po1] we showed how to deduce from the quantum Chevalley-Monk formula (8) the following quantum Pieri formulas for the quantum product of $e_{i}^{(k)}$ and $h_{i}^{(k)}$ with any Schubert class:

$$
\begin{aligned}
e_{i}^{(k)} * \sigma_{w} & =\mathcal{E}_{i}^{(k)}\left(\sigma_{w}\right), \\
h_{i}^{(k)} * \sigma_{w} & =\mathcal{H}_{i}^{(k)}\left(\sigma_{w}\right) .
\end{aligned}
$$


In a different form, the quantum Pieri formulas were earlier given by CiocanFontanine in $[\mathrm{C}-\mathrm{F}$. In the proof of these formulas given in [Po1] we only used the quadratic relations for the operators $\mathcal{T}_{i j}$ found by Fomin and Kirillov [F-K].

\section{PATH SCHubert POLYNOMials}

In this section we define path Schubert polynomials and establish their relation with the Gromov-Witten invariants for the flag manifold. These polynomials can be expressed in terms of paths in the quantum Bruhat graph. As a corollary, we obtain a combinatorial rule for all monomials $q^{d}$ that occur in the quantum product $\sigma_{u} * \sigma_{v}$ in terms of paths.

Let us define the operator $\mathcal{H}(y)$, acting on the space $\mathrm{QH}^{*}\left(\mathrm{Fl}_{n}\right) \otimes \mathbb{Z}\left[y_{1}, \ldots, y_{n}\right]$, as the following combination of quantum Pieri operators:

$$
\mathcal{H}(y)=\sum_{\beta \leq \delta} y^{\delta-\beta} \mathcal{H}_{\beta_{1}}^{(1)} \cdots \mathcal{H}_{\beta_{n-1}}^{(n-1)}
$$

where the sum is over nonnegative sequences $\beta=\left(\beta_{1}, \ldots, \beta_{n-1}\right)$ such that $\beta \leq \delta$ termwise, $\delta=(n-1, n-2, \ldots, 1)$, and $y^{\gamma}=y_{1}^{\gamma_{1}} y_{2}^{\gamma_{2}} \cdots y_{n-1}^{\gamma_{n-1}}$, for the termwise difference $\gamma=\beta-\delta$.

Let us define, for all pairs $u, v \in S_{n}$, the path Schubert polynomials $\mathfrak{S}_{u, v}=$ $\mathfrak{S}_{u, v}(y, q)$ in the polynomial ring $\mathbb{Z}\left[y_{1}, \ldots, y_{n}, q_{1}, \ldots, q_{n-1}\right]$ as the matrix elements of the operator $\mathcal{H}(y)$ in the basis of Schubert classes:

$$
\mathcal{H}(y): \sigma_{u} \longmapsto \sum_{v \in S_{n}} \mathfrak{S}_{u, v} \cdot \sigma_{v} .
$$

It follows from the definitions that the $\mathfrak{S}_{u, v}$ are polynomials with nonnegative integer coefficients.

The specialization of the $\mathfrak{S}_{u, v}$ for $q_{1}=\cdots=q_{n-1}=0$ are exactly the skew Schubert polynomials $\mathfrak{S}_{v / u} \in \mathbb{Z}\left[x_{1}, \ldots, x_{n}\right]$ recently introduced by Lenart and Sottile [L-S. We remark that the quantum Schubert polynomials $\mathfrak{S}_{w}^{q}$ from [FGP] cannot be obtained by a specialization of the path Schubert polynomials $\mathfrak{S}_{u, v}$. Indeed, all coefficients of $\mathfrak{S}_{u, v}$ are nonnegative, whereas $\mathfrak{S}_{w}^{q}$ may have negative coefficients. Nevertheless, as we will see, the polynomials $\mathfrak{S}_{u, v}$ are intimately related to the quantum cohomology $\mathrm{QH}^{*}\left(\mathrm{Fl}_{n}\right)$.

The definition of path Schubert polynomials $\mathfrak{S}_{u, v}$ can be reformulated in terms of paths in the quantum Bruhat graph. Let $\Gamma_{n}=\Gamma_{\Phi}$ be the quantum Bruhat graph for a type $A_{n-1}$ root system $\Phi$. The graph $\Gamma_{n}$ is a certain directed graph on the set of permutations in $S_{n}$. Each edge $e$ in $\Gamma_{n}$ is assigned a certain weight and a certain label. Two permutations $u$ and $v$ are connected by the directed edge $e: u \stackrel{i j}{\longrightarrow} v$ labeled $(i, j), 1 \leq i<j \leq n$, if and only if $v=u s_{i j}$ and one of the following two conditions is satisfied:

$$
\ell(v)=\ell(u)+1 \quad \text { or } \quad \ell(v)=\ell(u)+1-2(j-i) .
$$

If $\ell(v)=\ell(u)+1$, then the weight of edge $e$ equals 1 , and if $\ell(v)=\ell(u)+1-2(j-i)$, then the weight of edge $e$ equals $q_{i j}=q_{i} q_{i+1} \cdots q_{j-1}$. The weight of a directed path in the graph $\Gamma_{n}$ is the product of the weights of its edges.

Let us say that a directed path $u_{0} \stackrel{a_{1} b_{1}}{\longrightarrow} u_{1} \stackrel{a_{2} b_{2}}{\longrightarrow} \ldots \stackrel{a_{i} b_{i}}{\longrightarrow} u_{i}$ in the graph $\Gamma_{n}$ is $y_{k}^{i}$-admissible if $a_{1} \leq \cdots \leq a_{i} \leq k<b_{1}, \ldots, b_{i}$ and $b_{1}, \ldots, b_{i}$ are distinct; cf. the definition (10) of quantum Pieri operators $\mathcal{H}_{i}^{(k)}$. More generally, let us say that 
a path $P$ in the graph $\Gamma_{n}$ is $y^{\beta}$-admissible, for a monomial $y^{\beta}=y_{1}^{\beta_{1}} \ldots y_{n-1}^{\beta_{n-1}}$, if the path $P$ is a concatenation of $n-1$ paths $P=P_{1} \circ \cdots \circ P_{n-1}$ such that $P_{k}$ is $y_{k}^{\beta_{k}}$-admissible, for $k=1, \ldots, n-1$.

By definition, the path Schubert polynomial $\mathfrak{S}_{u, v}$ is equal to

$$
\mathfrak{S}_{u, v}=\sum_{\beta} y^{\delta-\beta} \sum_{P} \operatorname{weight}(P)
$$

where the second summation is over all $y^{\beta}$-admissible paths $P$ in the graph $\Gamma_{n}$ with initial vertex $u$ and terminal vertex $v$.

Every polynomial in the $y_{i}$ with $\operatorname{deg}_{y_{i}} \leq n-i$ can be expressed as a linear combination of the usual Schubert polynomials $\mathfrak{S}_{w}(y)=\mathfrak{S}_{w}\left(y_{1}, \ldots, y_{n}\right)$. The next theorem claims that the coefficients in such an expansion for path Schubert polynomials are exactly the Gromov-Witten invariants.

Theorem 4. For any $u, v \in S_{n}$, we have

$$
\mathfrak{S}_{u, w_{\mathrm{o}} v}(y, q)=\sum_{w, d}\left\langle\sigma_{u}, \sigma_{v}, \sigma_{w_{\mathrm{o}} w}\right\rangle_{d} q^{d} \mathfrak{S}_{w}(y),
$$

where the sum is over $w \in S_{n}$ and $d \in \mathbb{Z}_{\geq 0}^{n-1}$. In other words, the coefficient of $\mathfrak{S}_{w}$ in the Schubert expansion of $\mathfrak{S}_{u, w_{\mathrm{o}} v}$ is equal to the coefficient of the Schubert class $\sigma_{w}$ in the quantum product $\sigma_{u} * \sigma_{v}$.

This statement is a generalization to the quantum cohomology of a recent result by Lenart and Sottile [L-S, Theorem 1]. On the other hand, it is a flag manifold analog of [Po3, Theorem 6.3].

Theorem 4 implies, in particular, that

$$
\mathfrak{S}_{w, w_{\mathrm{o}}}(y, q)=\mathfrak{S}_{1, w_{\mathrm{o}} w}(y, q)=\mathfrak{S}_{w}(y)
$$

is the usual Schubert polynomial. Thus all $y^{\beta}$-admissible paths in $\Gamma_{n}$ with the initial vertex 1 or with the terminal vertex $w_{\mathrm{o}}$ have weight 1 , i.e., they are increasing saturated chains in the Bruhat order.

Following [L-S, Remark 6], note that both sides of the identity in Theorem 4 can be interpreted in terms of paths in the quantum Bruhat graph. It would be interesting to present a bijection between these two collections of paths. Such a bijection would provide a generalization of jeu de taquin to paths in the quantum Bruhat graph. Actually, such a bijection would imply a combinatorial interpretation for the Gromov-Witten invariants $\left\langle\sigma_{u}, \sigma_{v}, \sigma_{w_{o} w}\right\rangle_{d}$ similar to the jeu de taquin version of the classical Littlewood-Richardson rule.

Theorem 4 easily follows from the Cauchy identity (5), the elementary quantization rule (9), and the quantum Pieri formula (11).

Proof of Theorem 4 . Taking the image of the Cauchy identity (5), with the expanded left-hand side (6), in the ring $\mathrm{H}^{*}\left(\mathrm{Fl}_{n}\right) \otimes \mathbb{Z}\left[y_{1}, \ldots, y_{n}\right]$, we get

$$
\sum_{\beta} y^{\delta-\beta} e_{\beta_{1}}^{(n-1)} \cdots e_{\beta_{n-1}}^{(1)}=\sum_{w \in S_{n}} \mathfrak{S}_{w}(y) \cdot \sigma_{w w_{\mathrm{o}}} .
$$

According to the elementary quantization rule (9), we can write the following identity in the ring $\mathrm{QH}^{*}\left(\mathrm{Fl}_{n}\right) \otimes \mathbb{Z}\left[y_{1}, \ldots, y_{n}\right]$ :

$$
\sum_{\beta} y^{\delta-\beta} e_{\beta_{1}}^{(n-1)} * \cdots * e_{\beta_{n-1}}^{(1)}=\sum_{w \in S_{n}} \mathfrak{S}_{w}(y) \cdot \sigma_{w w_{\mathrm{o}}} .
$$


Applying the involution $\omega: \mathrm{QH}^{*}\left(\mathrm{Fl}_{n}\right) \rightarrow \mathrm{QH}^{*}\left(\mathrm{Fl}_{n}\right)$, we get

$$
\sum_{\beta} y^{\delta-\beta} h_{\beta_{1}}^{(1)} * \cdots * h_{\beta_{n-1}}^{(n-1)}=\sum_{w \in S_{n}} \mathfrak{S}_{w}(y) \cdot \sigma_{w_{\circ} w} .
$$

Since the operators of quantum multiplications by the $h_{i}^{(k)}$ are given by the quantum Pieri operators $\mathcal{H}_{i}^{(k)}$, the previous identity implies that

$$
\mathcal{H}(y): \sigma_{u} \mapsto \sum_{w \in S_{n}} \mathfrak{S}_{w}(y) \cdot\left(\sigma_{w_{\mathrm{o}} w} * \sigma_{u}\right) .
$$

The last formula is equivalent to the claim of the theorem.

Observe that the Cauchy identity that appears in the proof of Theorem 4 was an essential ingredient in the approach of Kirillov and Maeno [K-M to quantum Schubert calculus.

Theorem 4 implies a combinatorial description for the set of monomials $q^{d}$ in the quantum parameters that occur in the quantum product of two Schubert classes. Let us say that a directed path $u_{0} \stackrel{a_{1} b_{1}}{\longrightarrow} u_{1} \stackrel{a_{2} b_{2}}{\longrightarrow} \ldots \stackrel{a_{l} b_{l}}{\longrightarrow} u_{l}$ in the graph $\Gamma_{n}$ is admissible if there exists a sequence $k_{1} \leq \cdots \leq k_{l}$ such that $a_{i} \leq k_{i}<b_{i}$, for $i=1, \ldots, l$, and all pairs $\left(k_{1}, b_{1}\right), \ldots,\left(k_{l}, b_{l}\right)$ are distinct.

Corollary 5. For any $u, v \in S_{n}$ and $d \in \mathbb{Z}_{\geq 0}^{n-1}$, the monomial $q^{d}$ occurs in $\sigma_{u} * \sigma_{v}$ if and only if there exists an admissible path of weight $q^{d}$ from $u$ to $w_{\mathrm{o}} v$ in the graph $\Gamma_{n}$.

Proof. Let $\mathfrak{S}_{u, v, d} \in \mathbb{Z}\left[y_{1}, \ldots, y_{n}\right]$ be the coefficient of $q^{d}$ in the path Schubert polynomial $\mathfrak{S}_{u, v}$. By Theorem 4 a monomial $q^{d}$ occurs in $\sigma_{u} * \sigma_{v}$ if and only if $\mathfrak{S}_{u, w_{\mathrm{o}} v, d}$ is nonzero. On the other hand, by the definition of path Schubert polynomials, $\mathfrak{S}_{u, w_{\mathrm{o}} v, d}$ is nonzero if and only if there exists a path from $u$ to $w_{\mathrm{o}} v$ of weight $q^{d}$, which is $y^{\beta}$-admissible, for some $\beta$. Every $y^{\beta}$-admissible path is admissible and every admissible path reduces to a $y^{\beta}$-admissible path.

I would like to thank Misha Kogan, Chris Woodward, and Cristian Lenart for interesting discussions and helpful correspondence.

\section{REFERENCES}

[BFP] Francesco Brenti, Sergey Fomin, Alexander Postnikov: Mixed Bruhat operators and Yang-Baxter equations for Weyl groups, International Mathematics Research Notices 1999, no. 8, 419-441. MR2000e:20067

[C-F] IONuŢ Ciocan-Fontanine: On quantum cohomology rings of partial flag varieties, Duke Mathematical Journal 98 (1999), no. 3, 485-524. MR2000d:14058

[FGP] Sergey Fomin, Sergei Gelfand, Alexander Postnikov: Quantum Schubert polynomials, Journal of the American Mathematical Society 10 (1997), no. 3, 565-596. MR.98d:14063

[F-K] Sergey Fomin, Anatol N. Kirillov: Quadratic algebras, Dunkl elements, and Schubert calculus, in "Advances in Geometry," Progress in Mathematics 172 (1999), 147-182. MR,2001a:05152

[F-W] William Fulton, Christopher Woodward: On the quantum product of Schubert classes, e-print ArXiv math. AG/0112183.

[Hum] James E. Humphreys: Introduction to Lie Algebras and Representation Theory, second printing (revised), Graduate Texts in Mathematics 9, Springer-Verlag, New York, Berlin, 1978. MR $81 \mathrm{~b}: 17007$ 
[K-M] Anatol N. Kirillov, Toshiaki Maeno: Quantum double Schubert polynomials, quantum Schubert polynomials and Vafa-Intriligator formula, Discrete Mathematics 217 (2000), no. 1-3, 191-223. MR2001f:05161

[LSc] Alain Lascoux, Marcel-Paul Schützenberger: Polynômes de Schubert, Comptes Rendus des Séances de l'Académie des Sciences. Série I. Mathématique 294 (1982), no. 13, 447-450. MR83e:14039

[L-S] Cristian Lenart, Frank Sottile: Skew Schubert polynomials, Proceedings of the American Mathematical Society 131 (2003), no. 11, 3319-3328.

[Mac] I. G. Macdonald: Schubert polynomials, Cambridge University Press, Cambridge, 1991. MR 93d:05159

[Pet] Dale Peterson: Lectures on quantum cohomology of G/P, M.I.T., 1996.

[Po1] Alexander Postnikov: On a quantum version of Pieri's formula, in "Advances in Geometry," Progress in Mathematics 172 (1999), 371-383. MR99m:14096

[Po2] Alexander Postnikov: Symmetries of Gromov-Witten invariants, in "Advances in Algebraic Geometry Motivated by Physics," Contemporary Mathematics 276 (2001), 251-258. MR,2003c:14062

[Po3] Alexander Postnikov: Affine approach to quantum Schubert calculus, e-print ArXiv math.CO/0205165.

Department of Mathematics, Massachusetts institute of Technology, Cambridge, Massachusetts 02139

E-mail address: apost@math.mit.edu

URL: http://www.math-mit.edu/ apost/ 